\title{
EQUIVALENCE RELATIONS INDUCED BY ACTIONS OF POLISH GROUPS
}

\author{
SLAWOMIR SOLECKI
}

\begin{abstract}
We give an algebraic characterization of those sequences $\left(H_{n}\right)$ of countable abelian groups for which the equivalence relations induced by Borel (or, equivalently, continuous) actions of $H_{0} \times H_{1} \times H_{2} \times \cdots$ are Borel. In particular, the equivalence relations induced by Borel actions of $H^{\omega}, H$ countable abelian, are Borel iff $H \simeq \bigoplus_{p}\left(F_{p} \times \mathbb{Z}\left(p^{\infty}\right)^{n_{p}}\right)$, where $F_{p}$ is a finite $p$-group, $\mathbb{Z}\left(p^{\infty}\right)$ is the quasicyclic $p$-group, $n_{p} \in \omega$, and $p$ varies over the set of all primes. This answers a question of R. L. Sami by showing that there are Borel actions of Polish abelian groups inducing non-Borel equivalence relations. The theorem also shows that there exist non-locally compact abelian Polish groups all of whose Borel actions induce only Borel equivalence relations. In the process of proving the theorem we generalize a result of Makkai on the existence of group trees of arbitrary height.
\end{abstract}

\section{INTRODUCTION}

Let $G$ be a group acting on a set $X$. Put for $x, y \in X$

$$
x E_{G}^{X} y \Leftrightarrow \exists g \in G g x=y .
$$

Then $E_{G}^{X} \subset X \times X$ is an equivalence relation and is called the equivalence relation induced by the action of $G$ on $X$. If $G$ is Polish, $X$ is a standard Borel space, and the action of $G$ is Borel, then $E_{G}^{X}$ is $\Sigma_{1}^{1}$. If additionally $G$ is locally compact, then $E_{G}^{X}$ is Borel. By Silver's theorem, it follows that the topological Vaught conjecture holds in this case; i.e., the action of $G$ has either countably or "perfectly" many orbits. It was proved by R. L. Sami [S, Theorem 2.1] that the topological Vaught conjecture holds for Borel actions of abelian Polish groups. The proof, however, was different from the one in the locally compact case; in particular, it did not show that $E_{G}^{X}$ was Borel for $G$ Polish abelian.

The natural question was raised by Sami (see [S, p. 339]) whether $E_{G}^{X}$ is Borel for all Borel (or, equivalently, continuous if $X$ is a Polish space, see [BK]) actions of Polish abelian groups. We answer this question in the negative. We consider groups of the form $H_{0} \times H_{1} \times H_{2} \times \cdots$ where the $H_{n}$ 's are countable. Such groups are equipped with the product topology (each $H_{n}$ carrying the discrete topology) which is Polish and compatible with the group structure. We fully characterize those sequences $\left(H_{n}\right)$ of countable abelian groups for which

Received by the editors June 2, 1994.

1991 Mathematics Subject Classification. Primary 04A15; Secondary 20K25, 20K10, 20A05.

Key words and phrases. Actions of Polish groups, equivalence relation induced by an action. 
all Borel actions of $H_{0} \times H_{1} \times H_{2} \times \cdots$ induce Borel equivalence relations. This happens precisely when all but finitely many of the $H_{n}$ 's are torsion and, for each prime $p$, for all but finitely many $n$ 's the $p$-component of $H_{n}$ is of the form $F \times \mathbb{Z}\left(p^{\infty}\right)^{m}$, where $F$ is a finite $p$-group, $\mathbb{Z}\left(p^{\infty}\right)$ is the quasicyclic $p$-group (i.e., $\mathbb{Z}\left(p^{\infty}\right) \simeq\left\{z \in \mathbb{C}: \exists n z^{p^{n}}=1\right\}$ ), and $m \in \omega$. In particular, if $H_{n}=H, n \in \omega$, and $H$ is countable abelian, then all Borel actions of $H \times H \times H \times \cdots$ induce Borel equivalence relations iff $H \simeq \bigoplus_{p}\left(F_{p} \times \mathbb{Z}\left(p^{\infty}\right)^{n_{p}}\right)$, where $F_{p}$ is a finite abelian $p$-group, $n_{p} \in \omega$, and $p$ varies over the set of all primes. Thus, e.g., the group $\mathbb{Z} \times \mathbb{Z} \times \mathbb{Z} \times \cdots$ is abelian, Polish, and has a Borel action which induces a non-Borel equivalence relation. This answers Sami's question. On the other hand, $\mathbb{Z}\left(2^{\infty}\right) \times \mathbb{Z}\left(2^{\infty}\right) \times \mathbb{Z}\left(2^{\infty}\right) \times \cdots$ provides an interesting example of a Polish abelian group which is not locally compact but whose Borel actions induce only Borel equivalence relations. This shows that the implication " $G$ locally compact $\Rightarrow E_{G}^{X}$ Borel" cannot be reversed. Some results for non-abelian $H_{n}$ 's are also obtained.

Now, we state some definitions and establish notation. By $\omega$ we denote the set of all natural numbers $\{0,1,2, \ldots\}$. Ordinal numbers are identified with the set of their predecessors; in particular $n=\{0,1, \ldots, n-1\}$, for $n \in \omega$. By $\mathbb{Z}, \mathbb{Z}(p), \mathbb{Z}\left(p^{\infty}\right), p$ a prime, we denote the group of integers, the cyclic group with $p$ elements, and the quasicyclic $p$-group, respectively. By $e$ we denote the identity element of a group and by $\langle X\rangle$, for a subset $X$ of a group, the subgroup generated by $X$. We write $\langle h\rangle$ for $\langle\{h\}\rangle$. If $H$ is a group, $\bigoplus_{\omega} H$ stands for the direct sum of countably many copies of $H$. A group $H$ is called p-compact if for any decreasing sequence of groups $G_{k}<\mathbb{Z}(p) \times H$ with $\pi\left[G_{k}\right]=\mathbb{Z}(p)$, for each $k \in \omega$, we have $\pi\left[\bigcap_{k \in \omega} G_{k}\right]=\mathbb{Z}(p)$ where $\pi: \mathbb{Z}(p) \times H \rightarrow \mathbb{Z}(p)$ is the projection. If $H$ is an abelian group and $p$ is a prime, by the $p$-component of $H$ we mean the maximal $p$-subgroup of $H$.

For a sequence of sets $\left(H_{n}\right), n \in \omega$, we write

$$
H^{n}=H_{0} \times \cdots \times H_{n-1}, \quad H^{<\omega}=\bigcup_{n \in \omega} H^{n}, \quad \text { and } \quad H^{\omega}=H_{0} \times H_{1} \times \cdots .
$$

We also write $A^{\omega}$ for the product of infinitely many copies of $A$. If $x \in H^{\omega}$, put $\ln x=\omega$; if $\sigma \in H^{n}$, some $n \in \omega$, put $\operatorname{lh} \sigma=n$. For $\sigma \in H^{<\omega}$ and $x \in$ $H^{<\omega} \cup H^{\omega}$, we write $\sigma * x$ for the concatenation of $\sigma$ and $x$. If $x \in H^{<\omega} \cup H^{\omega}$ and $X \subset \omega$, we write $x \mid X$ for the unique element $y \in H^{<\omega} \cup H^{\omega}$ such that the domain of $y$ is $\omega$, if $X \cap \ln x$ is infinite, and $n$, if $X \cap \ln x$ is finite and has $n$ elements, and $y(i)=x$ (the $(i+1)$ th element of $X)$. A set $S \subset H^{<\omega}$ is called a tree on $\left(H_{n}\right)$ if $\sigma \in S$ implies $\sigma \mid n \in S$ for any $n<\ln (\sigma)$. If $S$ is a tree on $\left(H_{n}\right)$ and $\sigma \in H^{<\omega}$, put $S_{\sigma}=\left\{\tau \in H^{<\omega}: \sigma * \tau \in S\right\}$. For a tree $S$ on $\left(H_{n}\right), H_{n}$ countable, define $S^{\prime}=\{\sigma \in S: \exists \tau \in S \sigma \subset \tau, \sigma \neq \tau\}$. By transfinite induction define, for $\beta \in \omega_{1}, S^{0}=S$ and $S^{\beta}=\left(S^{\gamma}\right)^{\prime}$ if $\beta=\gamma+1$, and $S^{\beta}=\bigcap_{\gamma<\beta} S^{\gamma}$ if $\beta$ is limit. Put $\operatorname{ht}(S)=\min \left\{\beta: S^{\beta}=S^{\beta+1}\right\}$. For $\sigma \in H^{<\omega}$, put $r_{S}(\sigma)=\min \left\{\beta \in \omega_{1}: \sigma \notin S^{\beta}\right\}$ if there exists $\beta<\omega_{1}$ with $\sigma \notin S^{\beta}$, and $r_{S}(\sigma)=\omega_{1}$ otherwise. If there is no danger of confusion, we will omit the subscript in $r_{S}$. A tree on $\left(H_{n}\right)$ is well-founded if there is no sequence $\sigma_{i} \in S$, $i \in \omega$, such that $\sigma_{i} \subset \sigma_{i+1}$ and $\ln \left(\sigma_{i}\right) \rightarrow \infty$ as $i \rightarrow \infty$. Now, assume that the $H_{n}$ 's are groups. The identity element $(e, e, \ldots)$ of $H^{\omega}$ is denoted by $\vec{e}$. A tree $S$ on $\left(H_{n}\right)$ is called a coset tree if $S \cap H^{n}$ is a left coset of a subgroup of $H^{n}$ for any $n \in \omega$; i.e., if $\sigma_{1}, \sigma_{2}, \sigma_{3} \in S \cap H^{n}$, then $\sigma_{1} \sigma_{2}^{-1} \sigma_{3} \in S$. A coset 
tree $S$ is called a group tree if $S \cap H^{n}$ is a subgroup of $H^{n}$ for any $n \in \omega$. The notion of a group tree was introduced by Makkai in [M] and rediscovered by the author. We say that $\left(H_{n}\right)$ admits group (coset) trees of arbitrary height if, for any $\beta<\omega_{1}$, there is a group (coset) tree $T$ on $\left(H_{n}\right)$ with $\operatorname{ht}(T)>\beta$. Let $S$ be a coset tree on a sequence of groups $\left(H_{n}\right)$. Then for each $n \in \omega$ there is a unique subgroup $G_{n}$ of $H^{n}$ which $S \cap H^{n}$ is a coset of. We actually have $G_{n}=\sigma^{-1}\left(S \cap H^{n}\right)$ for any $\sigma \in S \cap H^{n}$. Define

$$
\alpha(S)=\bigcup_{n \in \omega} G_{n}
$$

Thus $\alpha(S)=\bigcup_{n \in \omega} \sigma_{n}^{-1}\left(S \cap H^{n}\right)$ where $\sigma_{n} \in S \cap H^{n}$ if $S \cap H^{n} \neq \varnothing$ and $\sigma_{n}=e$ otherwise. It is easy to see that $\alpha(S)$ is a group tree.

\section{MAIN RESUlts}

Theorem 1. Let $\left(H_{n}\right)$ be a sequence of countable abelian groups. Then the equivalence relation induced by any Borel action of $H^{\omega}$ is Borel iff, for all but finitely many $n, H_{n}$ is torsion, and for all primes $p$ for all but finitely many $n$ the p-component of $H_{n}$ is of the form $F \times \mathbb{Z}\left(p^{\infty}\right)^{k}$, where $k \in \omega$ and $F$ is a finite abelian p-group.

If $H$ is countable, abelian, and torsion, then $H=\bigoplus_{p} H_{p}$, where $p$ ranges over the set of all primes, and $H_{p}$ is the $p$-component of $H$ (see [F]). Thus we get the following corollary.

Corollary. Let $H$ be an abelian countable group. Then the equivalence relations induced by Borel actions of $H^{\omega}$ are Borel if and only if $H$ is isomorphic to $\bigoplus_{p}\left(F_{p} \times \mathbb{Z}\left(p^{\infty}\right)^{n_{p}}\right)$, where $p$ ranges over the set of all primes, $n_{p} \in \omega$, and $F_{p}$ is a finite abelian p-group.

For not necessarily abelian countable groups, we have the following version of one implication from Theorem 1. (The definition of $p$-compactness is formulated in the introduction.)

Theorem 2. Let $\left(H_{n}\right)$ be a sequence of countable groups. If for each prime $p$, for all but finitely many $n, H_{n}$ is p-compact, then the equivalence relations induced by Borel actions of $H^{\omega}$ are Borel.

It is an open question whether the converse of Theorem 2 holds. This would be a natural extension of Theorem 1, since, as we show in Lemma 9, a countable abelian group is $p$-compact iff it is torsion and its $p$-component has the form as in Theorem 1.

Some of the ingredients of the proofs are: the theorem of Becker and Kechris [BK] on the existence of universal actions, the structure theory for countable abelian groups, and a construction of group trees of arbitrary height. It turns out that both conditions in Theorem 1 are equivalent to $\left(H_{n}\right)$ not admitting group trees of arbitrary height (Lemma 12). This generalizes the known results that the sequence $\left(H_{n}\right), H_{n}=\mathbb{Z}$ for each $n \in \omega$, admits group trees of arbitrary height (Makkai [M, Lemma 2.6]), and that the sequence $\left(H_{n}\right), H_{n}=\bigoplus_{\omega} \mathbb{Z}(2)$ for each $n$, admits group trees of arbitrary height (Shelah [M, Appendix]). (See also [L, p. 979] for a proof of the latter result and its generalizations to groups which are direct sums of $\kappa$ many copies of $\mathbb{Z}(2)$ for certain cardinals $\kappa$.) The 
known proofs in the above two cases- $\mathbb{Z}$ and $\bigoplus_{\omega} \mathbb{Z}(2)$-were different from each other, and Makkai's construction for $\mathbb{Z}$ rested on Dirichlet's theorem on primes in arithmetic progressions. We present a construction (Lemma 10) that encompasses both these cases and is purely combinatorial.

Here is how Theorems 1 and 2 follow from the lemmas in Sections 3-5. In Section 3, we prove that all Borel actions of $H^{\omega},\left(H_{n}\right)$ a sequence of countable groups, induce Borel equivalence relations iff $\left(H_{n}\right)$ does not admit well-founded coset trees of arbitrary height (Lemma 2). In Section 4, we show that $\left(H_{n}\right)$ does not admit well-founded coset trees of arbitrary height iff it does not admit group trees of arbitrary height (Lemma 6). Then, in Section 5, we show that if for each prime $p$, for all but finitely many $n, H_{n}$ is $p$-compact, then $\left(H_{n}\right)$ does not admit group trees of arbitrary height (Lemma 8). This proves Theorem 2. Next, we prove that if $\left(H_{n}\right)$ is a sequence of abelian groups, then $\left(H_{n}\right)$ does not admit group trees of arbitrary height iff, for all but finitely many $n, H_{n}$ is torsion and, for all primes $p$, for all but finitely many $n$, the $p$-component of $H_{n}$ has the form as in Theorem 1 (Lemma 12). This proves Theorem 1.

\section{GROUP ACTIONS AND COSET TREES}

The following construction is from [BK]. Let $G$ be a Polish group. Consider $\mathscr{F}(G)$ the space of all closed subsets of $G$ with the Effros Borel structure, i.e., the Borel structure generated by sets of the form $\{F \in \mathscr{F}(G): F \cap V \neq \varnothing\}$ for $V \subset G$ open. Put $\mathscr{U}_{G}=\mathscr{F}(G)^{\omega}$, and define the following $G$-action on $\mathscr{U}_{G}:\left(g,\left(F_{n}\right)\right) \rightarrow\left(g F_{n}\right)$.

Theorem (Becker-Kechris [BK]). $\mathscr{U}_{G}$ with the above G-action is a universal Borel $G$ space, i.e., if $X$ is a standard Borel space on which $G$ acts by Borel automorphisms, then there is a Borel injection $\pi: X \rightarrow \mathscr{U}_{G}$ such that $\pi(g x)=$ $g \pi(x)$ for $g \in G$ and $x \in X$.

Let $X$ be a standard Borel $G$-space. Let $\pi: X \rightarrow \mathscr{U}_{G}$ be a Borel injection whose existence is guaranteed by the above theorem. Then, for $x, y \in X$, we have

$$
x E_{G}^{X} y \Leftrightarrow \pi(x) E_{G}^{\mathscr{L} / G} \pi(y) .
$$

This shows that the following corollary to the theorem above is true.

Lemma 1. Let $G$ be a Polish group. The relation induced by any Borel G-action is Borel iff the relation induced by the G-action on $\mathscr{U}_{G}$ is Borel.

Lemma 2. Let $\left(H_{n}\right)$ be a sequence of countable groups. The equivalence relation induced by any Borel $H^{\omega}$-action is Borel iff $\left(H_{n}\right)$ does not admit well-founded coset trees of arbitrary height.

Proof. Let $\mathscr{T}$ be the family of all trees on $\left(H_{n}\right)$. The set $\mathscr{T}$ is a Polish space with the topology generated by sets of the form $\{T \in \mathscr{T}: \sigma \in T\}$ and $\{T \in \mathscr{T}: \sigma \notin T\}$ for $\sigma \in H^{<\omega}$.

$(\Leftrightarrow)$ By Lemma 1 , it is enough to prove that the $H^{\omega}$-action on $\mathscr{U}_{H^{\omega}}$ induces a Borel relation. Let $\mathscr{T}_{p}$ be the family of all pruned trees on $\left(H_{n}\right)$, i.e., trees with no finite branches, with the topology inherited from $\mathscr{T}$. This topology makes $\mathscr{T}_{p}$ a Polish space. The mapping $\phi: \mathscr{T}_{p} \rightarrow \mathscr{F}\left(H^{\omega}\right)$ given by $\phi(T)=$ $\left\{x \in H^{\omega}: \forall n \in \omega \quad x \mid n \in T\right\}$ is a Borel isomorphism. For $x \in H^{\omega}$ and $T \in \mathscr{T}_{p}$ define

$$
x T=\left\{\sigma \in H^{<\omega}: \sigma \in x \mid m\left(T \cap H^{m}\right) \text { where } m=\operatorname{lh}(\sigma)\right\} .
$$


Then easily $x T \in \mathscr{T}_{p}$. Also $\phi(x T)=x \phi(T)$. Thus it is enough to check that the following action of $H^{\omega}$ on $\mathscr{T}_{p} \omega$ induces a Borel equivalence relation: $\left(x,\left(T_{n}\right)\right) \rightarrow\left(x T_{n}\right)$, for $x \in H^{\omega},\left(T_{n}\right) \in \mathscr{T}_{p}{ }^{\omega}$.

Now define $\Phi: \mathscr{T}_{p} \times \mathscr{T}_{p} \rightarrow \mathscr{T}$ by

$$
\Phi(T, S)=\left\{\sigma \in H^{<\omega}: T \cap H^{m}=\sigma\left(S \cap H^{m}\right) \text { where } m=\operatorname{lh}(\sigma)\right\} .
$$

Easily $\Phi(T, S)$ is a coset tree. Define the mapping $\Psi: \mathscr{T}_{p}{ }^{\omega} \times \mathscr{T}_{p}{ }^{\omega} \rightarrow \mathscr{T}$ by

$$
\Psi\left(\left(T_{n}\right),\left(S_{n}\right)\right)=\bigcap_{n \in \omega} \Phi\left(T_{n}, S_{n}\right) .
$$

Note that the intersection of a family of coset trees is a coset tree. Thus, for any $\left(T_{n}\right),\left(S_{n}\right) \in \mathscr{T}_{p}{ }^{\omega}, \Psi\left(\left(T_{n}\right),\left(S_{n}\right)\right)$ is a coset tree. Also note that

$$
\left(T_{n}\right) E_{H^{\omega}}^{\mathscr{Y}^{\omega}}\left(S_{n}\right) \Leftrightarrow \Psi\left(\left(T_{n}\right),\left(S_{n}\right)\right) \text { is not well-founded. }
$$

Indeed, if $\sigma_{0} \subset \sigma_{1} \subset \cdots, \operatorname{lh}\left(\sigma_{i}\right) \rightarrow \infty$, and $\sigma_{i} \in \Psi\left(\left(T_{n}\right),\left(S_{n}\right)\right)$, then $x S_{n}=T_{n}$ for each $n \in \omega$ where $x=\bigcup_{i \in \omega} \sigma_{i}$. If $x S_{n}=T_{n}$ for all $n \in \omega$ and some $x \in$ $H^{\omega}$, then $x \mid i \in \Psi\left(\left(T_{n}\right),\left(S_{n}\right)\right)$ and $\{x \mid i: i \in \omega\}$ witnesses that $\Psi\left(\left(T_{n}\right),\left(S_{n}\right)\right)$ is not well-founded. Clearly $\Psi$ is a Borel mapping. Thus, if we assume that there is $\beta \in \omega_{1}$ such that any well-founded coset tree on $\left(H_{n}\right)$ has height $<\beta$, we get

$$
\left(\mathscr{T}_{p} \times \mathscr{T}_{p}\right) \backslash E_{H^{\omega}}^{\mathscr{T}^{\omega}}=\Psi^{-1}(\{T \in \mathscr{T}: T \text { well-founded and ht }(T)<\beta\}) .
$$

But $\{T \in \mathscr{T}: T$ well-founded and $\mathrm{ht}(T)<\beta\}$ is Borel, whence $E_{H^{\omega}}^{\mathscr{T}^{\omega}}$ is Borel.

$\Leftrightarrow)$ Assume $\left(H_{n}\right)$ admits well-founded coset trees of arbitrary height. Define the following continuous action of $H^{\omega}$ on $\mathscr{T}$ :

$$
(x, T) \rightarrow x T=\left\{\sigma \in H^{<\omega}: \sigma \in x \mid m\left(T \cap H^{m}\right) \text { where } m=\operatorname{lh}(\sigma)\right\} .
$$

Define a Borel function $\Phi_{1}: \mathscr{T} \times \mathscr{T} \rightarrow \mathscr{T}$ by

$$
\Phi_{1}(T, S)=\left\{\sigma \in H^{<\omega}: \forall m \leq \operatorname{lh}(\sigma) T \cap H^{m}=\sigma \mid m\left(S \cap H^{m}\right)\right\} .
$$

Now, if $E_{H^{\omega}}^{\mathscr{S}}$ is Borel, $\Phi_{1}\left[(\mathscr{T} \times \mathscr{T}) \backslash E_{H^{\omega}}^{\mathscr{G}}\right]$ is $\Sigma_{1}^{1}$. Also $\Phi_{1}\left[(\mathscr{T} \times \mathscr{T}) \backslash E_{H^{\omega}}^{\mathscr{G}}\right] \subset$ $\{T \in \mathscr{T}: T$ is well-founded $\}$. Since $\{T \in \mathscr{T}: T$ is well-founded $\}$ is a $\Pi_{1}^{1}$ set and $T \rightarrow \mathrm{ht}(T)$ is a $\Pi_{1}^{1}$-norm on it, by the boundedness principle, there is $\beta \in$ $\omega_{1}$ such that, for any $T, S \in \mathscr{T}$, if $(T, S) \notin E_{H^{\omega}}^{\mathscr{G}}$, then $\operatorname{ht}\left(\Phi_{1}(T, S)\right)<\beta$. But note that if $T$ is a coset tree, then $\Phi_{1}(T, \alpha(T))=T$. Thus, for any wellfounded coset tree $T$ on $\left(H_{n}\right), \operatorname{ht}(T)=\operatorname{ht}(\Phi(T, \alpha(T)))<\beta$, a contradiction.

\section{COSET AND GROUP TREES}

The next several lemmas lead to a proof that the existence of well-founded coset trees of arbitrary height is equivalent to the existence of group trees of arbitrary height (Lemma 6). We will use a few times the easy fact that $\{r(\sigma): \sigma \in T\} \supset \mathrm{ht}(T)$ for any tree $T$ on $\left(H_{n}\right)$.

Lemma 3. Let $S$ be a coset tree. Then:

(i) $\alpha\left(S^{\prime}\right)=\alpha(S)^{\prime}$;

(ii) if $S^{\xi} \cap H^{k} \neq \varnothing$ for each $k \in \omega$, then $\alpha\left(S^{\xi}\right)=\alpha(S)^{\xi}$. 
Proof. To show (i), let $\sigma \in H^{n}$. Then $\sigma \in \alpha\left(S^{\prime}\right)$ implies that there are $\tau_{1}, \tau_{2} \in$ $S^{\prime}$ such that $\sigma=\tau_{1}^{-1} \tau_{2}$. Now we can find $g, h \in H_{n}$ with $\tau_{1} * g, \tau_{2} * h \in S$. But then $\sigma *\left(g^{-1} h\right)=\left(\tau_{1} * g\right)^{-1}\left(\tau_{2} * h\right) \in \alpha(S)$. Thus $\sigma \in \alpha(S)^{\prime}$. On the other hand, if $\sigma \in \alpha(S)^{\prime}$, then there are $g \in H_{n}$ and $\tau_{1}, \tau_{2} \in S$ with $\tau_{1}^{-1} \tau_{2}=\sigma * g$. But then $\sigma=\left(\tau_{1} \mid n\right)^{-1}\left(\tau_{2} \mid n\right)$ and $\tau_{1}\left|n, \tau_{2}\right| n \in S^{\prime}$, whence $\sigma \in \alpha\left(S^{\prime}\right)$.

Notice that if $S_{n} \supset S_{n+1}, n \in \omega$, are coset trees, and, for some $k \in \omega$, $\bigcap_{n \in \omega}\left(S_{n} \cap H^{k}\right) \neq \varnothing$, then $\alpha\left(\bigcap_{n \in \omega} S_{n}\right) \cap H^{k}=\bigcap_{n \in \omega} \alpha\left(S_{n}\right) \cap H^{k}$. To see this, pick $\sigma \in \bigcap_{n \in \omega} S_{n} \cap H^{k}$. Then

$$
\begin{aligned}
\alpha\left(\bigcap_{n \in \omega} S_{n}\right) \cap H^{k} & =\sigma^{-1}\left(\bigcap_{n \in \omega} S_{n} \cap H^{k}\right)=\bigcap_{n \in \omega} \sigma^{-1}\left(S_{n} \cap H^{k}\right) \\
& =\bigcap_{n \in \omega} \alpha\left(S_{n}\right) \cap H^{k} .
\end{aligned}
$$

Using (i) and the above observation, we get (ii) by transfinite induction.

Lemma 4. Let $T$ be a group tree. Let $\sigma_{n} \in H^{n}, n \in \omega$, be such that $\left(\sigma_{n+1} \mid n\right)^{-1} \sigma_{n}$ $\in T^{\beta}$ for some $\beta \in \omega_{1}$. Put $S=\bigcup_{n \in \omega} \sigma_{n}\left(T \cap H^{n}\right)$. Then $S$ is a coset tree, and for any $\xi \leq \beta$ we have $S^{\xi}=\bigcup_{n \in \omega} \sigma_{n}\left(T^{\xi} \cap H^{n}\right)$.

Proof. For $\xi \leq \beta$, define

$$
S^{(\xi)}=\bigcup_{n \in \omega} \sigma_{n}\left(T^{\xi} \cap H^{n}\right)
$$

In particular, $S^{(0)}=S$. First note that each $S^{(\xi)}$ is a coset tree. Indeed, if $m<n$, then $\left(\sigma_{n} \mid m\right)^{-1} \sigma_{m} \in T^{\xi}$. This follows easily by induction from our assumptions that it holds for $n=m+1$ and the fact that $T^{\xi}$ is a group tree. To check that $S^{(\xi)}$ is a tree, let $\tau \in T^{\xi} \cap H^{n}$. Then, for $m<n,\left(\sigma_{n} \tau\right) \mid m=$ $\left(\sigma_{n} \mid m\right)(\tau \mid m)=\sigma_{m}\left(\sigma_{m}^{-1}(\sigma \mid m)(\tau \mid m)\right) \in S^{(\xi)} \cap H^{m}$ since $\left(\sigma_{m}^{-1}\left(\sigma_{n} \mid m\right)\right)(\tau \mid m) \in T^{\xi} \cap$ $H^{m}$. Thus $S^{(\xi)}$ is a tree, and because of the way it was defined, it is a coset tree. It is obvious that $\alpha\left(S^{(\xi)}\right)=T^{\xi}$ and that $\sigma_{n} \in S^{(\xi)}$ for any $n \in \omega, \xi \leq \beta$.

Now, we show by induction that, for $\xi \leq \beta, \alpha\left(S^{\xi}\right)=T^{\xi}$ and $\sigma_{n} \in S^{\xi}$ for each $n \in \omega$. Both statements are true for $\xi=0$. If $\xi$ is the limit and $\sigma_{n} \in S^{\zeta}$ for all $\zeta<\xi$, then clearly $\sigma_{n} \in S^{\xi}$. By Lemma 3(ii), we also have $\alpha\left(S^{\xi}\right)=\alpha(S)^{\xi}=T^{\xi}$. If $\xi$ is a successor, say $\xi=\zeta+1$, then, by Lemma $3(\mathrm{i})$ and the induction hypothesis, we get $\alpha\left(S^{\zeta}\right)=\alpha\left(S^{\zeta}\right)^{\prime}=\left(T^{\zeta}\right)^{\prime}=T^{\xi}$. Since $\sigma_{n+1} \in S^{\zeta}, \sigma_{n+1} \mid n \in S^{\xi}$. Since $\left(\sigma_{n+1} \mid n\right)^{-1} \sigma_{n} \in T^{\beta} \subset T^{\xi}$, we have $\sigma_{n}=$ $\left(\sigma_{n+1} \mid n\right)\left(\left(\sigma_{n+1} \mid n\right)^{-1} \sigma_{n}\right) \in S^{\xi}$.

Thus $\alpha\left(S^{(\xi)}\right)=T^{\xi}=\alpha\left(S^{\xi}\right)$, i.e., for each $n \in \omega, S^{(\xi)} \cap H^{n}$ and $S^{\xi} \cap H^{n}$. are left cosets of the same subgroup of $H^{n}$. Also $\left(S^{(\xi)} \cap H^{n}\right) \cap\left(S^{\xi} \cap H^{n}\right) \neq \varnothing$, as $\sigma_{n}$ belongs to the intersection. Thus we get $S^{(\xi)} \cap H^{n}=S^{\xi} \cap H^{n}$ for each $n \in \omega$, i.e., $S^{(\xi)}=S^{\xi}$.

Lemma 5. Let $T$ be a group tree with $\mathrm{ht}(T)>\omega$. Then there exist $\sigma_{n} \in H^{n}$ such that:

(i) $\left(\sigma_{n+1} \mid n\right)^{-1} \sigma_{n} \in T$;

(ii) $\bigcup_{n \in \omega} \sigma_{n}\left(T \cap H^{n}\right)$ is a well-founded tree of height $<\omega \cdot 2$.

Proof. We start with the following observation. Let $K$ be a countable group and let $K_{n}, n \in \omega$, be a strictly decreasing sequence of subgroups of $K$. Then 
there exist $g_{n} \in K, n \in \omega$, such that $g_{n}^{-1} g_{n+1} \in K_{n}$ and $\bigcap_{n \in \omega} g_{n} K_{n}=\varnothing$. To see that this is true, enumerate $K=\left\{k_{n}: n \in \omega\right\}$ and pick $g_{n} \in K$ recursively so that $g_{n+1} K_{n+1} \subset g_{n} K_{n}$ and $k_{n} \notin g_{n+1} K_{n+1}$.

Now, assume that $T$ is a group tree and $\operatorname{ht}(T)>\omega$. Let $\sigma_{0}$ be such that $r\left(\sigma_{0}\right)=\omega$. Put $k_{0}=\ln \left(\sigma_{0}\right)+1$. Then $\left\{r(\sigma): \sigma \in T \cap H^{k_{0}}\right\} \cap \omega$ is cofinal in $\omega$. Let $p_{n}: H^{n} \rightarrow H^{k_{0}}, n>k_{0}$, denote the projection on the first $k_{0}$ coordinates. Since $\left\{\sigma \in H^{k_{0}}: r(\sigma) \geq m\right\}=p_{k_{0}+m}\left[T \cap H^{k_{0}+m}\right]$, there is an increasing sequence $k_{0}<m_{0}<m_{1}<m_{2}<\cdots$ such that $p_{m_{n+1}}\left[T \cap H^{m_{n+1}}\right] \neq p_{m_{n}}\left[T \cap H^{m_{n}}\right]$ and, obviously, $p_{m_{n+1}}\left[T \cap H^{m_{n+1}}\right] \subset p_{m_{n}}\left[T \cap H^{m_{n}}\right]$. Pick $\tau_{n} \in H^{k_{0}}, n \in \omega$, as in the preceding paragraph for $K_{n}=p_{m_{n}}\left[T \cap H^{m_{n}}\right]$, i.e.,

$$
\tau_{n+1}^{-1} \tau_{n} \in p_{m_{n}}\left[T \cap H^{m_{n}}\right] \text { and } \bigcap_{n \in \omega} \tau_{n}\left(p_{m_{n}}\left[T \cap H^{m_{n}}\right]\right)=\varnothing .
$$

We recursively construct $\sigma_{n} \in H^{n}, n \in \omega$, so that $\sigma_{m_{n}} \mid k_{0}=\tau_{n}$ and $\left(\sigma_{n+1} \mid n\right)^{-1} \sigma_{n} \in T$. First, find $\rho_{n} \in H^{m_{n}}$ so that $\rho_{n} \mid k_{0}=\tau_{n}$ and $\left(\rho_{n+1} \mid m_{n}\right)^{-1} \rho_{n}$ $\in T$. For $\rho_{0}$ take any extension of $\tau_{0}$ in $H^{m_{0}}$. Now assume $\rho_{n}$ has been constructed. Then $\tau_{n+1}^{-1}\left(\rho_{n} \mid k_{0}\right)=\tau_{n+1}^{-1} \tau_{n} \in p_{m_{n}}\left[T \cap H^{m_{n}}\right]$. Let $\sigma \in T \cap H^{m_{n}}$ be such that $\tau_{n+1}^{-1}\left(\rho_{n} \mid k_{0}\right)=\sigma \mid k_{0}$. Note that $\left(\rho_{n} \sigma^{-1}\right) \mid k_{0}=\tau_{n+1}$, and let $\rho_{n+1}$ be an arbitrary extension of $\rho_{n} \sigma^{-1}$ in $H^{m_{n+1}}$. Now, put $\sigma_{n}=\rho_{l} \mid n$ if $0 \leq n \leq m_{0}$ and $l=0$ or if $m_{l-1}<n \leq m_{l}$ and $l>0$.

We have $\sigma_{m_{n}}\left|k_{0}=\rho_{n}\right| k_{0}=\tau_{n}$. Also $\left(\sigma_{n+1} \mid n\right)^{-1} \sigma_{n} \in T$, i.e., (i) is easy to see. Put $S=\bigcup_{n \in \omega} \sigma_{n}\left(T \cap H^{n}\right)$. To check (ii), let $\sigma \in S \cap H^{k_{0}}$. Pick the unique $k \in \omega$ such that $\sigma \in \tau_{k}\left(p_{m_{k}}\left[T \cap H^{m_{k}}\right]\right) \backslash \tau_{k+1}\left(p_{m_{k+1}}\left[T \cap H^{m_{k+1}}\right]\right)$. Then for any $\sigma^{\prime} \in S$ with $\sigma^{\prime} \supset \sigma$, we have $l h \sigma^{\prime}<m_{k+1}$. Otherwise, $\sigma^{\prime} \in \sigma_{n}\left(T \cap H^{n}\right)$ for some $n \geq m_{k+1}$, whence $\sigma=p_{n}\left(\sigma^{\prime}\right) \in \tau_{n}\left(p_{n}\left[T \cap H^{n}\right]\right)$, a contradiction. Thus $r_{S}(\sigma)<\omega$ for any $\sigma \in S \cap H^{k_{0}}$. It follows that $S$ is well-founded and $\operatorname{ht}(S) \leq \omega+k_{0}$.

Lemma 6. Let $\left(H_{n}\right)$ be a sequence of countable groups. Then the following conditions are equivalent:

(i) $\left(H_{n}\right)$ admits well-founded coset trees of arbitrary height;

(ii) $\left(H_{n}\right)$ admits coset trees of arbitrary height;

(iii) $\left(H_{n}\right)$ admits group trees of arbitrary height.

Proof. (i) $\Rightarrow$ (ii) is obvious.

(ii) $\Rightarrow$ (iii). Note that if $S \subset T$ are coset trees and $S \neq T$, then $\alpha(S) \subset$ $\alpha(T)$ and $\alpha(S) \neq \alpha(T)$. To see this, pick $k \in \omega$ such that $S \cap H^{k} \neq T \cap H^{k}$ and $\sigma \in S \cap H^{k}$. Then $\alpha(S) \cap H^{k}=\sigma^{-1}\left(S \cap H^{k}\right) \neq \sigma^{-1}\left(T \cap H^{k}\right)=\alpha(T) \cap H^{k}$.

Now, let $S$ be a given coset tree. Define

$$
\gamma=\min \left\{\min \left\{\xi: \exists k S^{\xi} \cap H^{k}=\varnothing\right\}, \operatorname{ht}(S)\right\} .
$$

Then, by Lemma 3(ii) and the above observation, we have $\alpha(S)^{\xi}=\alpha\left(S^{\xi}\right) \neq$ $\alpha\left(S^{\zeta}\right)=\alpha(S)^{\zeta}$ for $\xi<\zeta<\gamma$, whence $\operatorname{ht}(\alpha(S)) \geq \gamma$. But it is easy to see that $\mathrm{ht}(S)<\gamma+\omega$. Thus (ii) $\Rightarrow$ (iii) is proved.

(iii) $\Rightarrow$ (i). Let $T$ be a group tree of height $>\beta+\omega$. We show that there is a well-founded coset tree of height $\geq \beta$. To this end consider $T^{\beta}$. Then $\operatorname{ht}\left(T^{\beta}\right)>\omega$. Apply Lemma 5 to $T^{\beta}$ to find $\sigma_{n} \in H^{n}, n \in \omega$, as in Lemma 5(i) and (ii). Put $S=\bigcup_{n \in \omega} \sigma_{n}\left(T \cap H^{n}\right)$. Then, by Lemma $4, S$ is 
a coset tree and $S^{\beta}=\bigcup_{n \in \omega} \sigma_{n}\left(T^{\beta} \cap H^{n}\right) \neq \varnothing$. By Lemma 5(ii), $S^{\beta+\omega \cdot 2}=$ $\left(\bigcup_{n \in \omega} \sigma_{n}\left(T^{\beta} \cap H^{n}\right)\right)^{\omega \cdot 2}=\varnothing$. Thus $S$ is a well-founded tree with $\mathrm{ht}(S) \geq \beta$.

\section{GROUP TREES AND ALGEBRAIC PROPERTIES OF GROUPS}

Lemma 7. Let $H$ be a countable group. If $H$ is not torsion, it is not p-compact for any prime $p$.

Proof. Clearly, if a subgroup of $H$ is not $p$-compact, neither is $H$. Thus it is enough to show that $\mathbb{Z}$ is not $p$-compact. This is witnessed by the following sequence of subgroups of $\mathbb{Z}(p) \times \mathbb{Z}$ :

$$
G_{k}=\left\{\left(m(p+1)^{k} \bmod p, m(p+1)^{k}\right): m \in \mathbb{Z}\right\}, \quad k \in \omega .
$$

Lemma 8. Let $\left(H_{n}\right)$ be a sequence of countable groups. If $\left(H_{n}\right)$ admits group trees of arbitrary height, then there exist a prime $p$ and infinitely many $n \in \omega$ such that $H_{n}$ is not p-compact.

Proof. If, for infinitely many $n \in \omega, H_{n}$ is not torsion, we are done by Lemma 7. Also, if $\left(H_{n}\right)$ admits group trees of arbitrary height, so does $\left(H_{n}\right)_{n \geq N}$ for any $N \in \omega$. This follows from Lemma 6 as soon as we notice that if $S$ is a coset tree on $\left(H_{n}\right)$ and $\sigma \in H^{N}$, then $S_{\sigma}$ is a coset tree on $\left(H_{n}\right)_{n \geq N}$, and that, given $\beta<\omega_{1}$, if $\mathrm{ht}(S)$ is large enough, then $\mathrm{ht}\left(S_{\sigma}\right)>\beta$ for some $\sigma \in H^{N}$. Thus, we can assume that $H_{n}$ is torsion for each $n$, and that there exists a group tree on $\left(H_{n}\right)$ of height $>\omega^{2}$.

Let $T$ be a group tree on $\left(H_{n}\right)$. Let $p$ be a prime. Assume $\sigma \in T \cap$ $H^{n}, r(\sigma)<\omega_{1}$, and the order of $\sigma$ is a power of $p$. Let $\beta<r(\sigma)$. Then there is $\tau \supset \sigma$ such that $r(\tau)=\beta$ and the order of $\tau$ is a power of $p$. To see this, let $\tau^{\prime} \supset \sigma, \tau^{\prime} \neq \sigma$ and $r\left(\tau^{\prime}\right) \geq \beta$. Let $l \in \omega$ be such that $p$ does not divide it and the order of $l \tau^{\prime}$ is a power of $p$. Since the order of $\sigma$ is a power of $p$, there is $l^{\prime} \in \omega$ such that $l^{\prime} l \sigma=\sigma$. Put $\tau_{1}=l^{\prime} l \tau^{\prime}$. Note that $\tau_{1} \supset \sigma$ and $\tau_{1} \neq \sigma$. Since, for any $\gamma \in \omega_{1}$ and $m \in \omega,\left\{\tau \in T \cap H^{m}: r(\tau) \geq \gamma\right\}$ is a subgroup of $H^{m}$ (this follows easily from the facts that $\left\{\tau \in T \cap H^{m}: r(\tau) \geq \gamma\right\}=T^{\gamma} \cap H^{m}$ and that $T^{\gamma}$ is a group tree), $r\left(\tau_{1}\right)=r\left(l^{\prime} l \tau^{\prime}\right) \geq r\left(\tau^{\prime}\right) \geq \beta$. If $r\left(\tau_{1}\right)=\beta$, we are done. If $r\left(\tau_{1}\right)>\beta$, we repeat the above construction and get $\tau_{2} \supset \tau_{1}, \tau_{2} \neq \tau_{1}$, whose order is a power of $p$ and $r\left(\tau_{2}\right) \geq \beta$. Again, if $r\left(\tau_{2}\right)=\beta$, we are done; otherwise we repeat the construction. Note that we cannot do it indefinitely, since then we would produce a sequence $\omega \subset \tau_{1} \subset \tau_{2} \subset \cdots, \tau_{m} \neq \tau_{m+1}$, whence $r(\sigma)=\omega_{1}$, a contradiction. Thus we must obtain $\tau_{m} \supset \sigma$ such that $r\left(\tau_{m}\right)=\beta$ and the order of $\tau_{m}$ is a power of $p$.

Next, notice that if $\tau \in T \cap H^{n}, r(\tau)$ is a limit, and the order of $\tau$ is a power of $p, p$ a prime, then $H_{n}$ is not $p$-compact. Indeed, let $\gamma_{k}, k \in \omega$, be a strictly increasing sequence of ordinals tending to $r(\tau)$. Put $G_{k}=\left\{\sigma \in T \cap H^{n+1}\right.$ : $\left.r(\sigma) \geq \gamma_{k}\right\}$. Let $\pi: H^{n+1} \rightarrow H^{n}$ be the projection. Notice that $\left(G_{k}\right)$ is a decreasing sequence of subgroups of $H^{n+1}$ and $\tau \in \bigcap_{k \in \omega} \pi\left[G_{k}\right] \backslash \pi\left[\bigcap_{k \in \sigma} G_{k}\right]$. Let $C=\langle\tau\rangle$. Then $C<H^{n}$ and $C \simeq \mathbb{Z}\left(p^{m}\right)$ for some $m \in \omega$. Put $G_{k}^{\prime}=$ $G_{k} \cap\left(C \times H_{n}\right)$. Let $\phi: C \rightarrow \mathbb{Z}(p)$ be a surjective homomorphism. Let $\Phi=$

$\phi \times$ id: $C \times H_{n} \rightarrow \mathbb{Z}(p) \times H_{n}$. Since $\Phi$ is finite-to-1, $\Phi\left[\bigcap_{k \in \omega} G_{k}^{\prime}\right]=\bigcap_{k \in \omega} \Phi\left[G_{k}^{\prime}\right]$. Note also that $\pi^{\prime} \circ \Phi=\phi \circ \pi$ where $\pi^{\prime}: \mathbb{Z}(p) \times H_{n} \rightarrow \mathbb{Z}(p)$ is the projection. 
Thus

$$
\phi\left[\pi\left[\bigcap_{k \in \omega} G_{k}^{\prime}\right]\right]=\pi^{\prime}\left[\bigcap_{k \in \omega} \Phi\left[G_{k}^{\prime}\right]\right] .
$$

But $\pi\left[\bigcap_{k \in \omega} G_{k}^{\prime}\right] \neq C$ whence $\pi\left[\bigcap_{k \in \omega} G_{k}^{\prime}\right] \subset \operatorname{ker}(\phi)$. Thus $\phi\left[\pi\left[\bigcap_{k \in \omega} G_{k}^{\prime}\right]\right]=$ $\{0\}$ and finally

$$
\pi^{\prime}\left[\bigcap_{k \in \omega} \Phi\left[G_{k}^{\prime}\right]\right]=\{0\}
$$

On the other hand,

$$
\bigcap_{k \in \omega} \pi^{\prime}\left[\Phi\left[G_{k}^{\prime}\right]\right]=\phi\left[\bigcap_{k \in \omega} \pi\left[G_{k}^{\prime}\right]\right]=\mathbb{Z}(p) .
$$

Thus the decreasing sequence of groups $\Phi\left[G_{k}^{\prime}\right], k \in \omega$, witnesses that $H_{n}$ is not $p$-compact.

Now, let $T$ be a group tree on $\left(H_{n}\right)$ with $\mathrm{ht}(T)>\omega^{2}$. There exists a prime $p$ and $\sigma \in T$ such that the order of $\sigma$ is a power of $p$ and $\omega^{2} \leq r(\sigma)<\omega_{1}$. To show this, first find $\tau \in T$ with $r(\tau)=\omega^{2}$. The group $G=\langle\tau\rangle$ is cyclic and finite. Thus there are $\sigma_{1}, \sigma_{2}, \ldots, \sigma_{m} \in T \cap H^{n}, n=l h(\tau)$, which commute with each other, their orders are powers of distinct primes and $\tau=\sigma_{0} \cdots \sigma_{m}$. Note that for each $0 \leq i \leq m$ there is $k \in \omega$ with $k \tau=\sigma_{i}$. Thus, since $\left\{\sigma \in T \cap H^{n}: r(\sigma) \geq \omega^{2}\right\}$ is a subgroup of $H^{n}, r\left(\sigma_{i}\right) \geq \omega^{2}$ for all $0 \leq i \leq m$. Also $\left\{\sigma \in T \cap H^{n}: r(\sigma) \geq \omega_{1}\right\}$ is a subgroup of $H^{n}$, thus there is $i$ such that $r\left(\sigma_{i}\right)<\omega_{i}$, and we are done.

Now, fix the prime $p$ and $\sigma \in T$ as above. Let $N \in \omega$. We show that there are more than $N$ numbers $n$ such that $H_{n}$ is not $p$-compact. Indeed, we can recursively produce $\tau_{0}, \tau_{1}, \ldots, \tau_{N} \in T$ so that $\sigma \subset \tau_{0}$ and $r\left(\tau_{0}\right)=\omega^{2}$, $\tau_{i} \subset \tau_{i+1}$, the order of each $\tau_{i}$ is a power of $p$, and $r\left(\tau_{i}\right)=\omega \cdot(N+1-i)$, $1 \leq i \leq N$. But then if we put $n_{i}=\ln \left(\tau_{i}\right)$, we get $n_{0}<n_{1}<\cdots<n_{N}$ and $H_{n_{i}}$ is not $p$-compact since $\omega^{2}$ and $\omega \cdot(N+1-i), 1 \leq i \leq N$, are limit.

In the following lemma, we essentially find all abelian countable groups which are $p$-compact.

Lemma 9. Let $H$ be an abelian countable group. Let $p$ be a prime. Then the following conditions are equivalent:

(i) $H$ is p-compact;

(ii) $H$ is torsion, and the $p$-component of $H$ is of the form $F \times \mathbb{Z}\left(p^{\infty}\right)^{n}$ where $F$ is a finite p-group and $n \in \omega$;

(iii) $H$ is torsion, and there is no surjective homomorphism mapping a subgroup of $H$ onto $\bigoplus_{\omega} \mathbb{Z}(p)$.

Proof. (ii) $\Rightarrow$ (i). Let $G_{k}<\mathbb{Z}(p) \times H, k \in \omega, G_{k+1}<G_{k}$, and $\pi\left[G_{k}\right]=$ $\mathbb{Z}(p)$ where $\pi: \mathbb{Z}(p) \times H \rightarrow \mathbb{Z}(p)$ is the projectin. Now, $H=H_{p} \times H^{\prime}$ and $G_{k}=\left(G_{k}\right)_{p} \times G_{k}^{\prime}$ where $H_{p}$ and $\left(G_{k}\right)_{p}$ are the $p$-components of $H$ and $G_{k}$, respectively, and the order of any element of $H^{\prime}$ or $G_{k}^{\prime}$ is not divisible by $p[\mathrm{~F}$, Theorem 8.4]. Clearly we have $\left(G_{k}\right)_{p}<\mathbb{Z}(p) \times H_{p}$. We say that a group fulfils the minimum condition if each strictly decreasing sequence of subgroups is finite. Since, as one can easily see, $\mathbb{Z}\left(p^{\infty}\right)$ and finite groups fulfil the minimum condition, and the property of fulfilling the minimum condition is preserved 
under taking finite products, $\mathbb{Z}(p) \times H_{p}$ fulfils the minimum condition. Thus there is $k_{0} \in \omega$ such that $\left(G_{k}\right)_{p}=\left(G_{k_{0}}\right)_{p}$ for $k \geq k_{0}$. But then

$$
\begin{aligned}
\pi\left[\bigcap_{k \in \omega} G_{k}\right] & =\pi\left[\bigcap_{k \in \omega}\left(G_{k}\right)_{p} \times \bigcap_{k \in \omega} G_{k}^{\prime}\right] \supset \pi\left[\left(G_{k_{0}}\right)_{p} \times\{0\}\right] \\
& =\pi\left[\left(G_{k_{0}}\right)_{p} \times G_{k_{0}}^{\prime}\right]=\pi\left[G_{k_{0}}\right]=\mathbb{Z}(p) .
\end{aligned}
$$

(i) $\Rightarrow$ (iii). By Lemma 7, $H$ is torsion. Note that if $F_{1}$ can be mapped by a homomorphism onto $F_{2}, F_{1}, F_{2}$ groups, and $F_{2}$ is not $p$-compact, then $F_{1}$ is not $p$-compact either. Indeed, let $\phi: F_{1} \rightarrow F_{2}$ be a surjective homomorphism, and let the sequence $\left(G_{k}\right)$ of subgroups of $\mathbb{Z}(p) \times F_{2}$ witness that $F_{2}$ is not $p$-compact, then

$$
G_{k}^{\prime}=\left\{(m, g) \in \mathbb{Z}(p) \times F_{1}:(m, \phi(g)) \in G_{k}\right\}
$$

witness that $F_{1}$ is not $p$-compact. Thus to prove that $H$ is not $p$-compact, assuming (iii) fails, it is enough to show that $\bigoplus_{\omega} \mathbb{Z}(p)$ is not $p$-compact. Let $\left\{e_{i}: i \in \omega\right\}$ be an independent set generating $\bigoplus_{\omega} \mathbb{Z}(p)$. Let us fix a sequence of sets $X_{k} \subset \omega, k \in \omega$, such that $X_{k+1} \subset X_{k}$ and $\bigcap_{k \in \omega} X_{k}=\varnothing$. Define $G_{k}<\mathbb{Z}(p) \times \bigoplus_{\omega} \mathbb{Z}(p)$ by

$$
G_{k}=\left\langle\left\{\left(m, m e_{i}\right): i \in X_{k}, m \in \mathbb{Z}(p)\right\}\right\rangle .
$$

Then $\left(G_{k}\right)$ witnesses that $\bigoplus_{\omega} \mathbb{Z}(p)$ is not $p$-compact.

(iii) $\Rightarrow$ (ii). Assume (iii). Let $H_{p}$ be the $p$-component of $H$. Let $H_{p}^{1}=$ $\bigcap_{n \in \omega} n H_{p}$ be its first Ulm group. If $H_{p} / H_{p}^{1}$ is infinite, then

$$
H_{p} / H_{p}^{1} \simeq \bigoplus_{m \in \omega} \mathbb{Z}\left(p^{n_{m}}\right)
$$

for a sequence $n_{m} \in \omega \backslash\{0\}$ [F, Theorem 17.2 and remarks on p. 155]. Thus $H_{p} / H_{p}^{1}$, and hence $H_{p}$, can be mapped homomorphically onto $\bigoplus_{\omega} \mathbb{Z}(p)$. Therefore $H_{p} / H_{p}^{1}$ is finite. Put $F=H_{p} / H_{p}^{1}$. But then $H_{p}^{1}$ is divisible [F, Lemma 37.2] and $H_{p} \simeq F \times H_{p}^{1}[\mathrm{~F}$, Theorem 21.2]. Now, by [F, Theorem 23.1], either $H_{p}^{1} \simeq \mathbb{Z}\left(p^{\infty}\right)^{n}$, for some $n \in \omega$, and we are done, or $H_{p}^{1} \simeq \bigoplus_{\omega} \mathbb{Z}\left(p^{\infty}\right)$. But in the latter case $H_{p}^{1}$, and hence $H$, contain an isomorphic copy of $\bigoplus_{\omega} \mathbb{Z}(p)$, a contradiction

Remark. (In this remark the notation and terminology follow [F].) One can give other characterizations of $p$-compactness among countable torsion abelian groups. For example $p$-compactness of $H$ is equivalent to the following conditions:

(iv) the $p$-component of $H$ fulfills the minimum condition;

(v) for any finite $p$-group $F<H$ the $p$-rank of $H / F$ is finite. Obviously (ii) $\Rightarrow$ (iv), and (iv) $\Rightarrow$ (i) as in the proof of (ii) $\Rightarrow$ (i). Now, assuming (iv) and noticing that a homomorphic image of a group fulfilling the minimum condition fulfills the minimum condition, we get that the $p$ component of $H / F, F<H$ finite, fulfills the minimum condition. This obviously implies that its $p$-rank is finite. Thus (iv) $\Rightarrow(v)$. To see (v) $\Rightarrow$ (ii), let $H_{p}$ be the $p$-component of $H$. Let $\tau$ be its Ulm type. First note that if $\tau=$ $\gamma+1$, for some $\gamma$, then $H_{p}^{\gamma} / H_{p}^{\tau}$ is finite. Otherwise, $r_{p}\left(H_{p}^{\gamma} / H_{p}^{\tau}\right)=\infty$, and since $H_{p} \simeq H_{p} / H_{p}^{\tau} \times H_{p}^{\tau}$, we get $r_{p}\left(H_{p}\right)=\infty$. Now, we claim that $\tau$ is neither a limit 
ordinal nor a successor of a limit ordinal. Otherwise, using the above observation there is a sequence of groups $G_{n}<H_{p} / H_{p}^{\tau}, n \in \omega$, such that $G_{n+1}<G_{n}$, $G_{n+1} \neq G_{n}$ and $\bigcap_{n \in \omega} G_{n}$ is finite. Put $G=\bigcap_{n \in \omega} G_{n}$. Then we can pick recursively $g_{k} \in H_{p} / H_{p}^{\tau}$ so that $p g_{k} \in G$ and for each $k$ there is an $n$ with $g_{k} \in G_{n}$ and $g_{i} \notin G_{n}$ for $i<k$. Then clearly the image of $\left\{g_{k}: k \in \omega\right\}$ under the natural homomorphism $H_{p} / H_{p}^{\tau} \rightarrow\left(H_{p} / H_{p}^{\tau}\right) / G$ is infinite independent. Again, since $H_{p} \simeq H_{p} / H_{p}^{\tau} \times H_{p}^{\tau}, r_{p}\left(H / G^{\prime}\right)=r_{p}\left(H_{p} / G^{\prime}\right)=\infty$ for some finite $p$-group $G^{\prime}$. Next, notice that $\tau$ is not of the form $\gamma+2$ because in this case $H_{p}^{\gamma+1} / H_{p}^{\tau}$ is finite and $r_{p}\left(H_{p}^{\gamma} / H_{p}^{\gamma+1}\right)=\infty$ whence $r_{p}\left(\left(H_{p}^{\gamma} / H_{p}^{\tau}\right) /\left(H_{p}^{\gamma+1} / H_{p}^{\tau}\right)\right)=\infty$. And as before $r_{p}\left(H / G^{\prime}\right)=\infty$ for some finite $p$-group $G^{\prime}$. Thus $\tau \leq 1$, and if $\tau=1$, then $H_{p} / H_{p}^{1}$ is finite. If $\tau=0, H_{p}$ is divisible, and since $r_{p}\left(H_{p}\right)<\infty$, there is $n \in \omega$ with $H_{p} \simeq \mathbb{Z}\left(p^{\infty}\right)^{n}$. If $\tau=1$, put $F=H_{p} / H_{p}^{1}$. Then $H_{p} \simeq F \times H_{p}^{1}$, $F$ finite, $H_{p}^{1}$ divisible. Since $r_{p}\left(H_{p}^{1}\right)<\infty$, there is $n \in \omega$ with $H_{p}^{1} \simeq \mathbb{Z}\left(p^{\infty}\right)^{n}$.

Now, we make a technical definition useful in proving the existence of group trees of arbitrary height. An abelian countable group $H$ is called manageable if there exist two decreasing sequences of subgroups $\left(G_{n}^{0}\right),\left(G_{n}^{1}\right)$ with $\bigcap_{n \in \omega} G_{n}^{i}=$ $\{e\}$, for $i=0,1$, and a homomorphism $\phi: H \times H \rightarrow H$ such that $\phi\left[G_{n}^{0} \times G_{n}^{1}\right]=$ $H$ for any $n \in \omega$.

Lemma 10. Let $H$ be a countable abelian group. If $H$ is manageable, then $\left(H_{n}\right)$, where $H_{n}=H$ for each $n \in \omega$, admits group trees of arbitrary height.

Proof. Fix two decreasing sequences of subgroups $\left(G_{n}^{0}\right)$ and $\left(G_{n}^{1}\right)$ and a homomorphism $\phi$ as in the definition of manageability. For each ordinal $\beta<\omega_{1}$, we produce a group tree $T_{\beta}$ such that:

- if $\beta=\gamma+1$, then $T_{\beta}^{\gamma} \cap H=H$ and $\forall h \in H\left(h \neq e \Rightarrow\left(T_{\beta}\right)_{h}\right.$ is well-founded);

- if $\beta$ is limit, then $\forall \gamma<\beta \exists n \in \omega\left(T_{\beta}^{\gamma} \cap H^{2} \supset G_{n}^{0} \times G_{n}^{1}\right)$ and $\forall \sigma \in H^{2}(\sigma \neq$ $(e, e) \Rightarrow\left(T_{\beta}\right)_{\sigma}$ is well-founded).

Then clearly $\omega_{1}>r_{T_{\beta}}(h) \geq \beta$ for any $h \in H \backslash\{e\}$ in the first case, and for any $\gamma<\beta, \omega_{1}>r_{T_{\beta}}(\sigma) \geq \gamma$ for some $\sigma \in H^{2} \backslash\{(e, e)\}$ in the latter. Thus $\operatorname{ht}\left(T_{\beta}\right) \geq \beta$ for any $\beta \in \omega_{1}$.

Put $T_{0}=\{\vec{e}\}$ and $T_{1}=H \cup\{\vec{e}\}$. Assume $T_{\gamma}$ has been defined for all $\gamma<\beta$. If $\beta=\gamma+1$ and $\gamma$ is a successor, put

$$
T_{\beta}=\{\varnothing\} \cup H \cup\left\{\sigma(0) * \sigma: \sigma \in T_{\gamma}, \operatorname{lh} \sigma \geq 1\right\} .
$$

If $\beta=\gamma+1$ and $\gamma$ is a limit, put

$T_{\beta}=$ the tree generated by $\left\{\phi(\sigma(0), \sigma(1)) * \sigma: \sigma \in T_{\gamma}\right.$, lh $\left.\sigma \geq 2\right\}$.

Checking that the $T_{\beta}$ 's work is straightforward. Now, assume $\beta$ is a limit ordinal. Note that it is enough to construct two group trees $S_{0}$ and $S_{1}$ such that there is an increasing sequence $\gamma_{n} \rightarrow \beta$ with $S_{0}^{\gamma_{n}} \cap H \supset G_{n}^{0}$ and $S_{1}^{\gamma_{n}} \cap H \supset G_{n}^{1}$ and $\forall h \in H\left(h \neq e \Rightarrow\left(S_{0}\right)_{h}\right.$ and $\left(S_{1}\right)_{h}$ are well-founded). If $S_{0}$ and $S_{1}$ are defined, let

$$
T_{\beta}=\left\{\sigma \in H^{<\omega}: \sigma \mid\{2 k: k \in \omega\} \in S_{0} \text { and } \sigma \mid\{2 k+1: k \in \omega\} \in S_{1}\right\} .
$$

We will define a group tree $S=S_{0}$ as above; the construction of $S_{1}$ is analogous. Put $G_{n}^{0}=G_{n}$. Fix an increasing sequence of successors $\gamma_{n} \rightarrow$ 
$\beta, n \in \omega$. Find pairwise disjoint infinite sets $X_{n}, n \in \omega$, with $\bigcup_{n \in \omega} X_{n}=\omega$. Let

$$
\begin{aligned}
& R_{n}=\{\varnothing\} \cup\left\{h * \sigma: h \in G_{n}, \sigma\left|X_{n} \in T_{\gamma_{n}}, \sigma\right|\left(\omega \backslash X_{n}\right) \subset \vec{e},\right. \text { and } \\
&\text { if } \left.l h \sigma>\min X_{n}, \text { then } h=\left(\sigma \mid X_{n}\right)(0)\right\} .
\end{aligned}
$$

Note that each $R_{n}$ is a group tree. Define

$$
S=\bigcup_{k \in \omega}\left\langle H^{k} \cap \bigcup_{n \in \omega} R_{n}\right\rangle .
$$

Easily $S$ is a group tree. To see $S^{\gamma_{n}} \cap H \supset G_{n}$, just notice that, for each $h \in G_{n}, r_{T_{\gamma_{n}}}(h) \geq \gamma_{n}$, and there is a monotone 1-to-1 mapping $\psi:\left(T_{\gamma_{n}}\right)_{h} \rightarrow S$ defined by $\psi(\sigma)=h * \tau$, where $\tau \in H^{<\omega}$ is maximal such that $\tau \mid X_{n}=h * \sigma$ and $\tau \mid\left(\omega \backslash X_{n}\right) \subset \vec{e}$. To show that $(S)_{h}$ is well-founded for $h \in H \backslash\{e\}$, fix $h \in H$ with $h \neq e$, and assume towards a contradiction that $h * x$ is an infinite branch through $S$ for some $x \in H^{\omega}$. Find $n \in \omega$ with $h \notin G_{n}$. Let $k \in \omega$ be such that $k \cap X_{i} \neq \varnothing$ for $i \in n$. Put $\tau=x \mid k$ and $n_{i}=\min X_{i}$ for $i \in n$. If $\tau\left(n_{i_{0}}\right) \neq e$ for some $i_{0} \in n$, notice that $x \mid X_{i_{0}}$ is an infinite branch through $T_{\gamma_{i_{0}}}$ with $\left(x \mid X_{i_{0}}\right)(0) \neq e$ which contradicts the inductive assumption. Thus we can assume that $\tau\left(n_{i}\right)=e$ for all $i \in n$. Then, since the $R_{i}$ 's are group trees, $h * \tau=\sigma \cdot \prod_{i \in n}\left(h_{i} * \tau_{i}\right)$ for some $\sigma \in G_{n} \times H^{k}$ with $\sigma\left(n_{i}\right)=e$ and some $h_{i} * \tau_{i} \in R_{i} \cap H^{k+1}$. By the definition of $R_{i}, h_{i}=\tau_{i}\left(n_{i}\right)=\tau\left(n_{i}\right)=e$. Thus $h=\sigma(0) \in G_{n}$, a contradiction.

Lemma 11. Let $\left(H_{n}\right)$ be a sequence of countable groups. Then $\left(H_{n}\right)$ admits group trees of arbitrary height if either of the following conditions holds.

(i) There exists a sequence $n_{0}<n_{1}<\cdots$ such that $\left(H_{n_{k}}\right)$ admits group trees of arbitrary height.

(ii) For each $n, G_{n}$ is a homomorphic image of a subgroup of $H_{n}$, and $\left(G_{n}\right)$ admits group trees of arbitrary height.

Proof. (i) Let $T$ be a group tree on $\left(H_{n_{k}}\right)$. Define $\bar{T}$ a group tree on $\left(H_{n}\right)$ as follows:

$$
\sigma \in \bar{T} \quad \text { iff } \sigma \mid X \in T \text { and } \sigma|(\omega \backslash X)=\vec{e}|(\omega \backslash X)
$$

where $X=\left\{n_{k}: k \in \omega\right\}$. Then $\operatorname{ht}(\bar{T}) \geq \operatorname{ht}(T)$.

(ii) Fix $H_{n}^{\prime}<H_{n}$ and surjective homomorphisms $\phi_{n}: H_{n}^{\prime} \rightarrow G_{n}$. Let $T$ be a group tree on $\left(G_{n}\right)$. Define $\bar{T}$ a group tree on $\left(H_{n}\right)$ as follows:

$$
\sigma \in \bar{T} \text { iff } \forall k<\operatorname{lh} \sigma\left(\sigma(k) \in H_{n}^{\prime} \text { and }\left(\phi_{0}(\sigma(0)), \ldots, \phi_{k}(\sigma(k))\right) \in T\right) .
$$

Then $\operatorname{ht}(\bar{T}) \geq \operatorname{ht}(T)$.

Lemma 12. Let $\left(H_{n}\right)$ be a sequence of countable abelian groups. Then $\left(H_{n}\right)$ does not admit group trees of arbitrary height iff $H_{n}$ is torsion for all but finitely many $n$, and for each prime $p$, for all but finitely many $n$ the $p$-component of $H_{n}$ is of the form $F \times \mathbb{Z}\left(p^{\infty}\right)^{k}$, where $F$ is a finite $p$-group, $k \in \omega$.

Proof. The implication $\Leftarrow$ follows from Lemmas 8 and 9. To see $\Rightarrow$, assume the conclusion does not hold. Then either there exist infinitely many $n$ such that $H_{n}$ contains an isomorphic copy of $\mathbb{Z}$ or, by Lemma 9 , there exist a prime $p$ and infinitely many $n$ such that a subgroup of $H_{n}$ can be mapped homomorphically onto $\bigoplus_{\omega} \mathbb{Z}(p)$. Thus, by Lemma 11 , it is enough to show 
that $\left(H_{n}\right)$, where $H_{n}=\mathbb{Z}$ for each $n$ or $H_{n}=\bigoplus_{\omega} \mathbb{Z}(p)$ for each $n$, admits group trees of arbitrary height.

By Lemma 10 , it suffices to prove that $\mathbb{Z}$ and $\bigoplus_{\omega} \mathbb{Z}(p)$ are manageable. For $\mathbb{Z}$, put $G_{n}^{0}=\left\langle 2^{n}\right\rangle, G_{n}^{1}=\left\langle 3^{n}\right\rangle$. Define $\phi: \mathbb{Z} \times \mathbb{Z} \rightarrow \mathbb{Z}$ by $\phi(m, l)=m+l$. For $\bigoplus_{\omega} \mathbb{Z}(p)$, fix an infinite independent set $\left\{e_{i}: i \in \omega\right\}$ generating $\bigoplus_{\omega} \mathbb{Z}(p)$. Find a decreasing sequence of nonempty sets $X_{n} \subset \omega, n \in \omega$, such that $\bigcap_{n \in \omega} X_{n}=$ $\varnothing$. Put $G_{n}^{0}=\left\langle\left\{e_{i}: i \in X_{n}\right\}\right\rangle$ and $G_{n}^{1}=\{e\}$. Fix a function $f: \omega \rightarrow \omega$ so that, for any $n, m \in \omega, f^{-1}(m) \cap X_{n} \neq \varnothing$. Define $\phi^{\prime}: \bigoplus_{\omega} \mathbb{Z}(p) \rightarrow \bigoplus_{\omega} \mathbb{Z}(p)$ to be the unique homomorphism extending $\phi^{\prime}\left(e_{i}\right)=e_{f(i)}$. Let $\phi: \bigoplus_{\omega} \mathbb{Z}(p) \times$ $\bigoplus_{\omega} \mathbb{Z}(p) \rightarrow \bigoplus_{\omega} \mathbb{Z}(p)$ be the composition of the projection to the first coordinate with $\phi^{\prime}$.

\section{ACKNOWLEDGMENTS}

I would like to thank Professor A. S. Kechris for valuable discussions and for pointing out the relevance of the papers [L] and [M].

\section{REFERENCES}

[BK] H. Becker and A. S. Kechris, Borel actions of Polish groups, Bull. Amer. Math. Soc. (N.S.) 28 (1993), 334-341.

[F] L. Fuchs, Infinite abelian groups, vols. I and II, Academic Press, New York and London, 1970, 1973.

[L] D. Lascar, Why some people are excited by Vaught's conjecture, J. Symbolic Logic 50 (1985), 973-981.

[M] M. Makkai, An example concerning Scott heights, J. Symbolic Logic 46 (1981), 301-318.

[S] R. L. Sami, Polish group actions and the Vaught conjecture, Trans. Amer. Math. Soc. 341 (1994), 335-353.

Department of Mathematics 253-37, California Institute of Technology, Pasadena, California 91125

Current address: Department of Mathematics, University of California, Los Angeles, California 90095

E-mail address: solecki@cco.caltech.edu 